\title{
Resistance thermometers based on the germanium films
}

\author{
V. F. Mitin \\ Institute of Semiconductor Physics, National Academy of Sciences of Ukraine, Kyiv, 252028, Ukraine, \\ phone: (380)442655939, e-mail: mitin@msens.kiev.ua; mitin@isp.kiev.ua
}

\begin{abstract}
The latest achievements in the field of development of resistance thermometers based on the germanium films on gallium arsenide are presented and summarized. Basic models of Ge film thermometers, which cover the temperature range from 0.02 to $500 \mathrm{~K}$, are considered. Characteristics of the thermometers in high magnetic fields and under the action of ionizing irradiation (neutrons and gamma-rays) are presented.
\end{abstract}

Keywords: thermometers, temperature sensors, germanium films.

Paper received 16.03.99; revised manuscript received 27.05.99; accepted for publication 27.05.99.

\section{Introduction}

At present, a great variety of devices are available for measurements of cryogenic temperatures. Because of the diversity of objects requiring temperature measurements and a broad range of performance requirements, there are many types of sensors based on different physical effects and fabricated from different materials [1, 2]. The intense development of new cryogenic technologies, and also of the physics of low and ultra-low temperatures makes it necessary to reduce the dimensions of the devices, to extend the temperature range toward the ultralow temperatures, to minimize the effect of the magnetic field on the temperature values measured, and to increase their radiation stability. It is especially important when the thermometers are used in the modern systems of highenergy physics, whose operation is associated with high radiation levels, high magnetic fields, and low temperatures [3].

At present, the most commonly used cryogenic resistance thermometers are germanium (from 0.05 to $100 \mathrm{~K}$ ), carbonic (from 1.4 to $325 \mathrm{~K}$ ), metal-oxide (from 70 to $325 \mathrm{~K}$ ) and platinum (from 30 to $800 \mathrm{~K}$ ) thermoresistors fabricated on bulk materials, and also film thermoresistors based on iron-rhodium (from 1.4 to $325 \mathrm{~K}$ ), rhutenium oxide (from 0.05 to $300 \mathrm{~K}$ ), and cernox (from 0.3 to $325 \mathrm{~K}$ )
$[2,4]$.

Radically new opportunities for development of cryogenic resistance thermometers arise if sensor elements are based on semiconding epitaxial films grown on insulating substrates. The thin-film technology is compatible with the integrated technology of semiconductor device fabrication. Employment of the batch approach commonly used in microelectronics to fabricate cryogenic temperature sensors makes it possible to reduce their dimensions and cost, to improve their technical characteristics, to expand the application range, and also allows the sensors to be fabricated both in the form of discrete devices and as components of integrated circuits.

The present work summarizes the latest achievements in the field of fabrication of the resistance thermometers based on the films of germanium on gallium arsenide. In this paper, the main models of small-size resistance thermometers are presented, covering the range of temperatures from 0.02 to $500 \mathrm{~K}$. Discussed are the problems of production of thermosensitive germanium films, designs of the sensor elements and of a small-size case, and the characteristics of thermometers in the temperature range from $25 \mathrm{mK}$ to $500 \mathrm{~K}$ in high magnetic fields and under the influence of ionizing radiation (neutrons and gamma-ray photons). 


\section{Ge films on GaAs as a thermosensitive material for thermometers}

The Ge/GaAs heterosystem consists of elementary and binary semiconductors. The difference of the lattice parameters for Ge and GaAs is as small as $0.08 \%$. Due to this fact, the intrinsic mechanical stresses at the film-substrate interface are relatively weak. In this sense, Ge and GaAs is an ideal heteropair. The coefficients of linear expansion of both materials are practically the same in a wide temperature range. A small mismatch of the lattice parameters and the absence of significant thermal stresses in the heterosystem create favorable conditions for epitaxial growth of films with a perfect structure.

A plenty of papers studying the Ge/GaAs interface and Ge films indicate that the technology of heterostructure formation essentially affects the structure, electrical and optical properties of Ge films [5-9].

The processes of defect generation in Ge/GaAs heterostructures are essentially different depending on which material is used as a substrate and which one is deposited as a film. When GaAs is deposited onto a Ge substrate, most defects at the film-substrate interface and in the GaAs film are associated with the antiphase disorder. If $\mathrm{Ge}$ is deposition on a GaAs substrate, the properties of the film are most affected by diffusion of $\mathrm{Ga}$ and As atoms. $\mathrm{Ga}$ is known to behave in $\mathrm{Ge}$ as an acceptor, and As as a donor impurity. Therefore, the diffusion processes near the film-substrate interface essentially affect the electrical properties of Ge films. The concentration of $\mathrm{Ga}$ and $\mathrm{As}$ in Ge films depends essentially on the technological conditions of heterostructure formation, such as the substrate temperature during the film deposition, rate of deposition and quality of the GaAs surface prior to deposition. The structure of the GaAs surface can be changed by various techniques, for example, by thermal treatment, ion bombardment, or chemical treatment. All these factors change, first of all, the content of Ga, As and oxygen at the surface and in the nearsurface GaAs layer and affect also the crystal structure of the surface layer.

Depending on the technological conditions, the impurity concentration in Ge films may vary from $10^{17}$ to $10^{21} \mathrm{~cm}^{-3}$. It is possible to produce both $\mathrm{p}$ - and n-type films with a different degree of compensation. Depending on the formation conditions, the resistivity (free carrier concentration) in Ge films at room temperature may vary from $10^{-3}$ to $140 \mathrm{Ohmcm}\left(10^{14}\right.$ to $\left.5 \times 10^{20} \mathrm{~cm}^{-3}\right)[6,9]$.

Thus, the electrical properties of Ge films on GaAs are controlled by two factors: Ga and As diffusion from the GaAs substrate during the film growth, and the degree of structural quality of the film. The crystalline structure of the films is mainly determined by the substrate temperature during the film deposition. An improvement of the structural quality changes the temperature dependencies of all characteristics of the film, such as the electrical conductance, carrier concentration and mobility [9].
To obtain Ge films with electrical properties suitable for fabrication of a sensor, one should find the technological conditions of film deposition resulting in a spectrum of impurity states in the film that would yield the appropriate electrical parameters. Thus, by varying the technological conditions of Ge deposition, one can obtain the films with required electrical and optical properties and fabricate a variety of physical sensors (of temperature, deformation, pressure, magnetic field, and optical illumination) [5, 9-11].

\section{Types of resistance thermometers based on ger- manium films, their design and characteristics}

A number of types of the resistance thermometers based on Ge films having different characteristics were designed for application in different temperature ranges [9-14]. The thermosensitive films were obtained by the techniques of thermal evaporation of $\mathrm{Ge}$ in vacuum $\left(2 \times 10^{-4} \mathrm{~Pa}\right)$ on semi-insulating GaAs substrates. The thermosensitive components were fabricated using the microelectronic technology.

Fig. 1 shows a typical design of the thermosensitive element of the resistance thermometer. The sizes of the sensitive element for different sensor models were $0,3 \times 0,3 \times 0,2 \mathrm{~mm}, 0,5 \times 0,5 \times 0,2 \mathrm{~mm}$ and $1,0 \times 1,0 \times 0,2 \mathrm{~mm}$. The pattern of the sensor was made using a photolitograghic technique. Using different photomasks, it is possible to change the pattern of the sensor's active region and to design thermometers with the resistance values required by the customers.

It is seen from Fig. 1 that the sensitive element is a multilayer structure. The basis of the sensor is a thermosensitive Ge film on a semi-insulating GaAs substrate. To form electrical contacts on Ge, thin AuGe (eutectic) and Au layers were subsequently deposited, with the total thickness of about $0.3 \mu \mathrm{m}$. In some cases, a thin Mo layer was formed

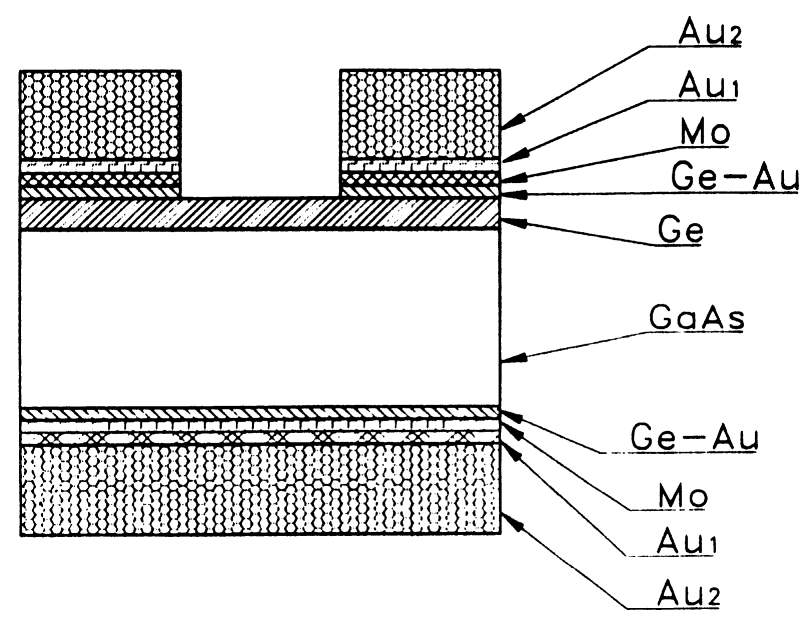

Fig. 1. Design of the thermosensitive element 


\section{F. Mitin: Resistance thermometers based on the germanium films ...}

between the layers of AuGe and Au. The metal films were deposited in vacuum using the magnetron sputtering technique. Such a multilayer system should provide a good electrical contact to $\mathrm{Ge}$ in a wide temperature range, from ultra-low to room temperatures. The Mo layer was used as a barrier for Au diffusion into Ge.

The contact leads were fabricated by thermocompression or pulse welding of a thin $(6 \mu \mathrm{m})$ gold strip with a thickness of about $150-200 \mu \mathrm{m}$, or of a gold wire with a diameter of $30 \mu \mathrm{m}$. To ensure the reliability of microwelding, an additional Au layer was deposited using the electrochemical deposition technique.

Fig. 2 shows the basic design of thermometer cases.
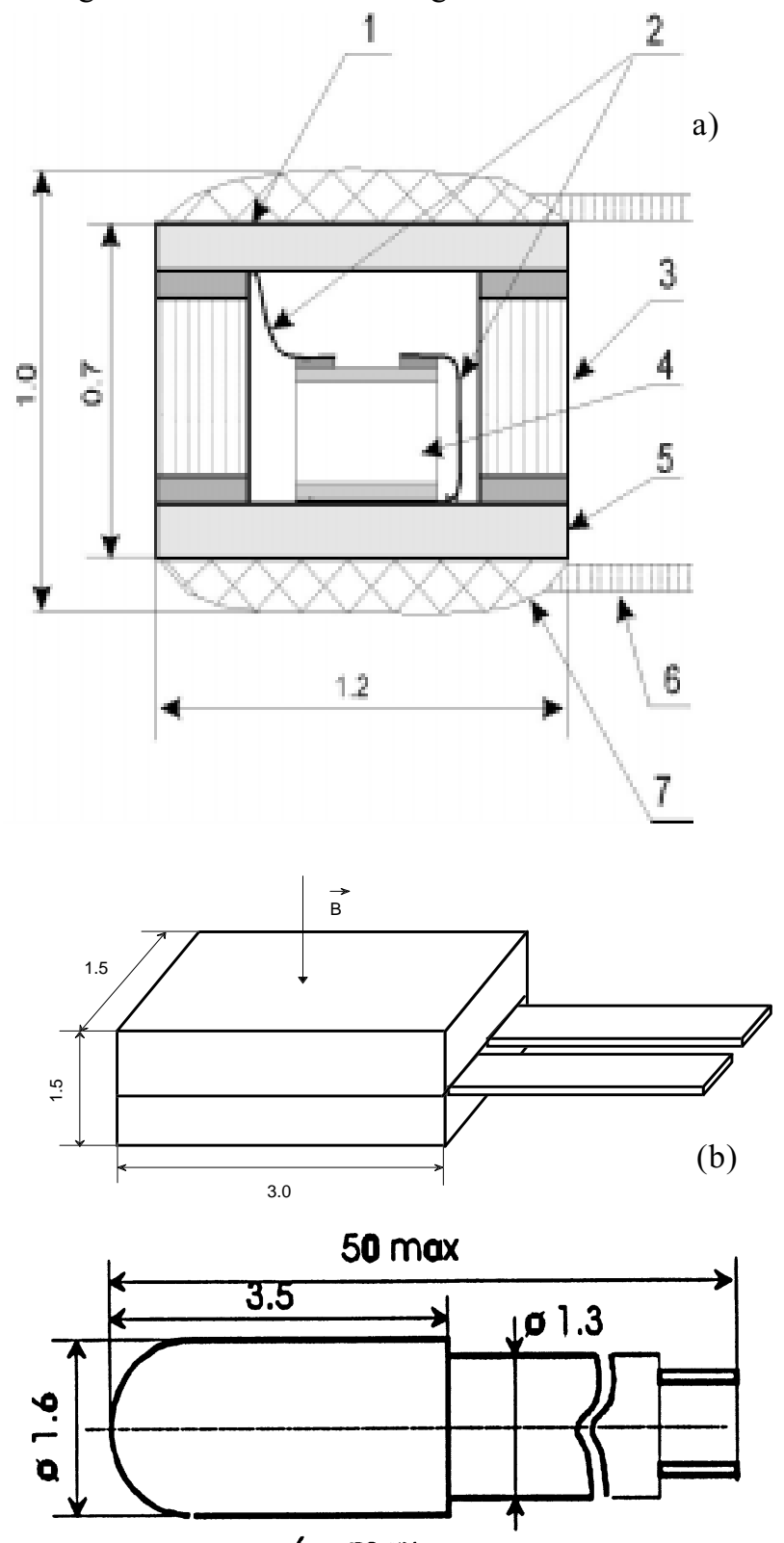

6. $\max$

(c)

Fig. 2. Designs and dimensions (in $\mathrm{mm}$ ) of thermometer cases: (a) metal-ruby case: (1) and (5) - copper disks; (2) - golden strip; (3) - ruby cylinder; (4) - sensitive element; (6) - copper wire; (7) - tin; (b) ceramic case; (c) metal case.
The smallest one is shown in Fig. 2a and consists of a ruby cylinder and two copper disks plated with a thin $\mathrm{Au}$ layer. The faces of the ruby cylinder are also covered with a thin layer of Au. The hermetic mounting of the disks to the ruby cylinder was made by thermodiffusion welding between the Au layers. The sensitive element was placed on one of the copper disks by thermodiffusion welding. The outer electrical leads were made using a copper wire with a diameter of $50 \mu \mathrm{m}$ soldered by tin to the copper disks. Including the thickness of the outer electrical tin and the lead solder, the overall dimensions of the thermometer were $\varnothing 1,2 \times 1,0 \mathrm{~mm}$.

It should be noted that stability and reproducibility of the characteristics of cryogenic thermometers based on Ge films depend essentially on the quality of fabrication technology of the sensor, on micropackaging and the type of the thermometer case. It is known that the most severe mode of the thermometer operation is its rapid (several seconds) cooling and heating, when the device is subjected to thermal shock. We have carried out the preliminary investigations of the strength of cases of different design and of the stability of the characteristics of thermometers in conditions of rapid cooling and heating (cyclic thermal shocks) in the range 77-300 $\mathrm{K}$ and 4.2-300 K. The results of preliminary investigations have shown that the case presented in the Fig. 1 a has the most reliable design, and the sensors in these cases have the most stable characteristics. Preliminary investigations indicate that after approximately 100 cycles of thermal shocks the stability of thermometer readings at $77.4 \mathrm{~K}$ is better than $\pm 25 \mathrm{mK}$, and at $4.2 \mathrm{~K}$ is better than $\pm 10 \mathrm{mK}$. At slow (tens of minutes and longer) cooling-heating cycles the reproducibility of thermometer characteristics is much better.

Consider the thermometric characteristics of some basic models of germanium film resistance thermometers.

\subsection{Thermometer for the temperature range from $25 \mathrm{mK}$ to $300 \mathrm{~K}$ (model TTR-1D)}

The most appropriate characteristics for production of lowtemperature resistance thermometers were found in Ge films with the thickness of about $1.5 \mu \mathrm{m}$. Films were single crystalline and with $p$-type of conductivity. The free carrier concentration in the films determined from the Hall measurements at $298 \mathrm{~K}$ was around $8.3 \times 10^{17} \mathrm{~cm}^{-3}$, mobility $-214 \mathrm{~cm}^{2} \mathrm{~V}^{-1} \mathrm{~s}^{-1}$ and resistivity $-3.5 \times 10^{-2} \mathrm{Ohm} \mathrm{cm}$. The sensitive elements with dimensions $0.3 \times 0.3 \times 0.2 \mathrm{~mm}^{3}$ were placed into the hermetic cases shown in Fig. 2 a.

Fig. 3 shows the temperature dependencies of resistance $R$ and sensitivity $|S|=|d R / d \mathrm{~T}|$ for thermometers TTR-1D in the temperature range from $25 \mathrm{mK}$ to $300 \mathrm{~K}$. In the same figure the thermometric characteristics in the range 1.7-300 $\mathrm{K}$ are shown for the batch of thermometers fabricated from the same Ge/GaAs structure. The differences of the temperature dependencies of resistance for different sensors is related to nonuniformity of $\mathrm{Ge}$ films. It should be noted that the application of batch 


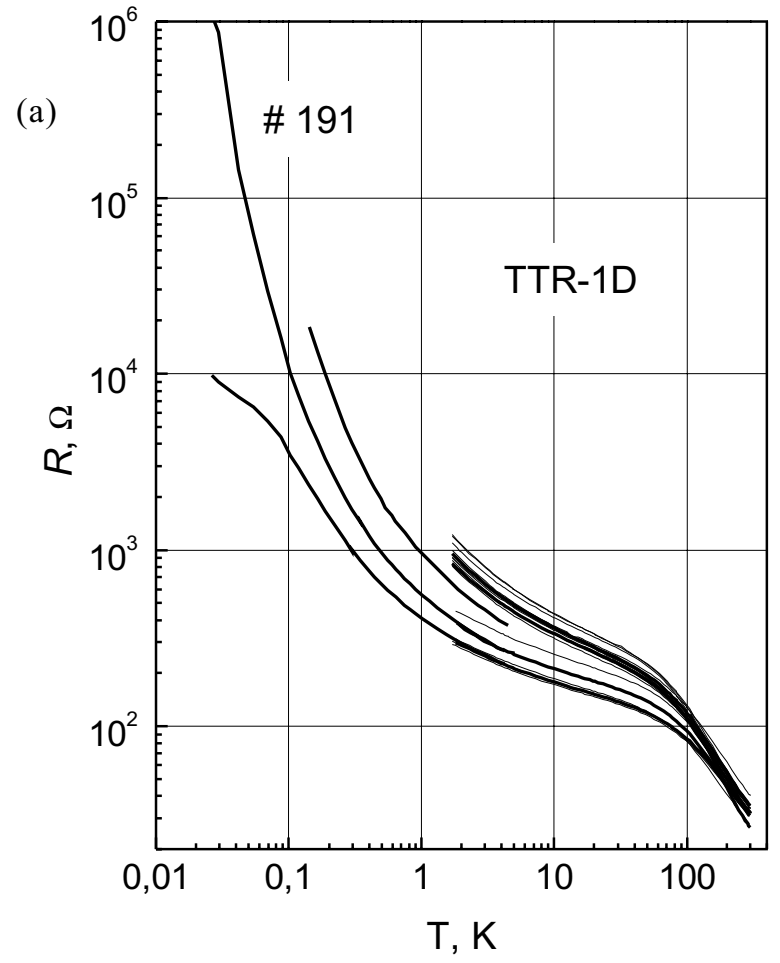

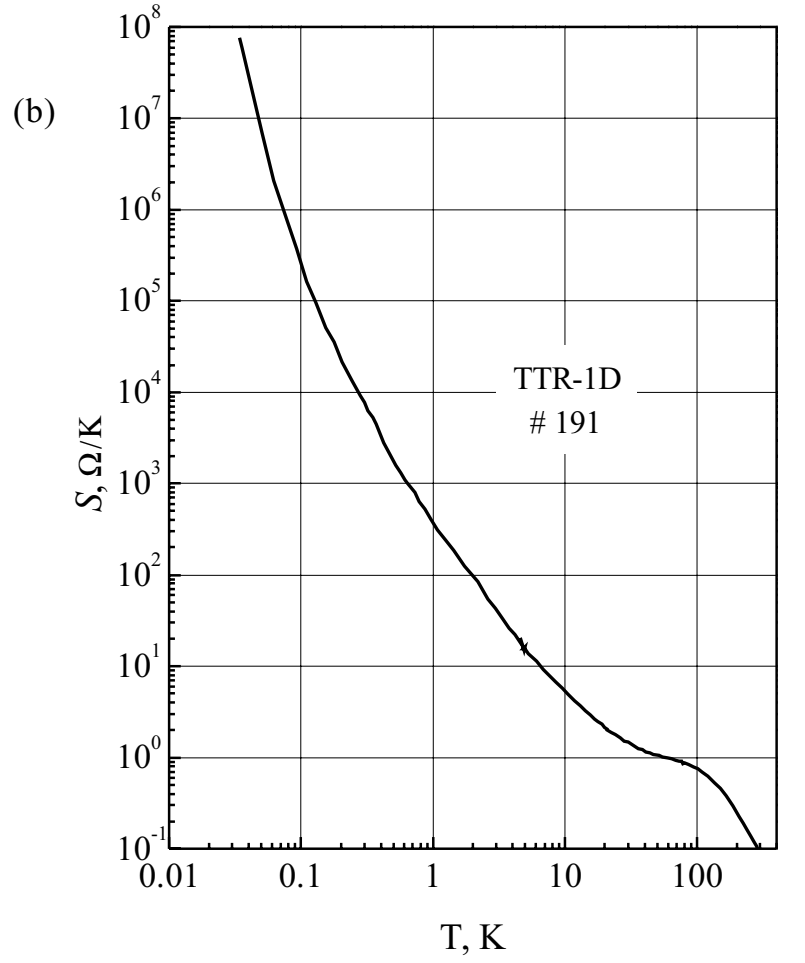

Fig. 3. Temperature dependences of resistance (a) and sensitivity (b) for TTR-1D thermometer.

techniques of microelectronics to sensor fabrication allows to fabricate up to 2000 temperature sensors from a single $\mathrm{Ge} / \mathrm{GaAs}$ wafer $30 \mathrm{~mm}$ in diameter. Thermometric characteristics of the sensors fabricated from the same wafer can differ, which is connected with the micro-nonuniformities of the germanium films. Nonuniformity of electrical properties of films is due to many technological factors and depends, first of all, on the quality of film deposition technology. From a great number of sensors fabricated using the single wafer, it is possible to select batches (some tens of items) of sensors with the same thermometric characteristics, which could be interchanged.

The investigations of volt-ampere characteristics of thermometers have shown that at the temperature $4.2 \mathrm{~K}$, when the thermometer is placed in the liquid helium, its volt-ampere characteristic is symmetrical and linear with an accuracy better than $0.1 \%$ up to the current values of about $100 \mu \mathrm{A}$. At higher currents the essential reduction of the resistance is observed due to self-heating of the thermometer.

3.2. Thermometers for the temperature range 1.5$300 \mathrm{~K}$ and $0.5-300 \mathrm{~K}$ (models TTR-1A and TTR-1B)

The sizes of the sensors of the TTR-1A thermometer are $0.5 \times 0.5 \times 0.2 \mathrm{~mm}$, and those for the TTR-1B thermome- ter were $1.0 \times 1.0 \times 0.2 \mathrm{~mm}$. The sensors were placed into cases shown in Fig. 2 b. Figure 4 shows the temperature dependencies of resistance $R$ and sensitivity $S$ for thermometers TTR-1A and TTR-1B. Figure 5 presents curves for the batch of TTR-1B thermometers in the range from 1.7 to $300 \mathrm{~K}$ fabricated from the same thermosensitive Ge/GaAs structure. Dimensionless sensitivity $\left|S_{d}\right|=|(\mathrm{T} / R)(d R / d \mathrm{~T})|$ for the cryogenic thermometers TTR-1A, TTR-1B and TTR-1D is shown in Fig. 6.

Current-voltage characteristic of the TTR-1B thermometer at $4.2 \mathrm{~K}$ is symmetrical and linear up to a current value of about $20 \mu \mathrm{A}$, and that for TTR-1A thermometer - up to $1 \mu \mathrm{A}$. At higher currents the reduction of the resistance is observed due to self-heating of the thermometer.

\subsection{Thermometers for the temperature range 77- $400 \mathrm{~K}$ and 200-500 K (models TTR-2 and TTR-3)}

The thermometers for operation in the range above $77 \mathrm{~K}$ were fabricated on the basis of heavily doped and strongly compensated germanium films [6, 9]. Electrical transport in such films was controlled by percolation, which provided a high thermal sensitivity in the range of relatively high temperatures. The sensor sizes of thermometers were $0.5 \times 0.5 \times 0.2 \mathrm{~mm}^{3}$. Main characteristics of the thermometer are presented in Fig. 7, and the case design is shown in Fig. 2 c. 
V. F. Mitin: Resistance thermometers based on the germanium films ...
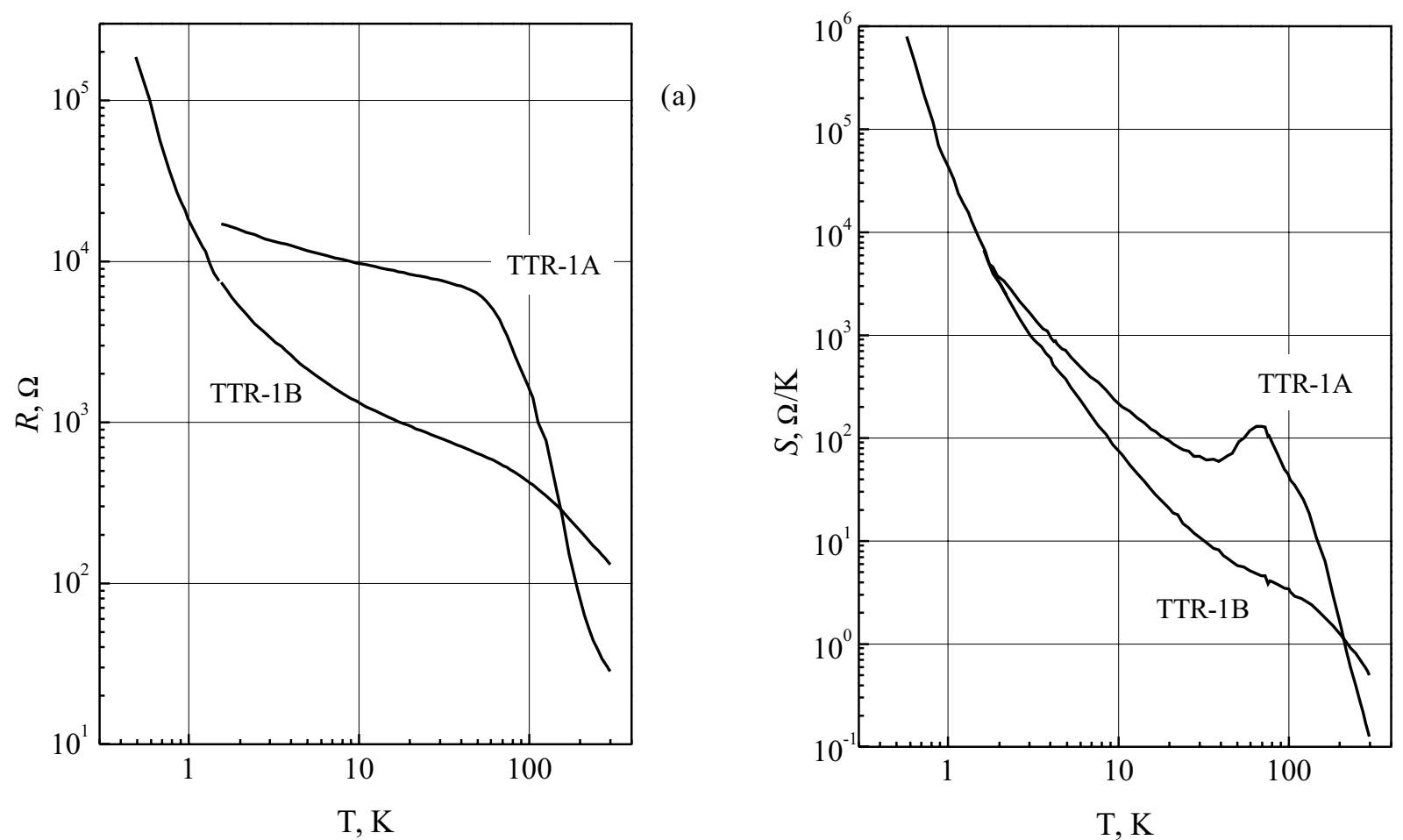

(b)

Fig. 4. Temperature dependences of resistance (a) and sensitivity (b) for TTR-1A and TTR-1B thermometers.

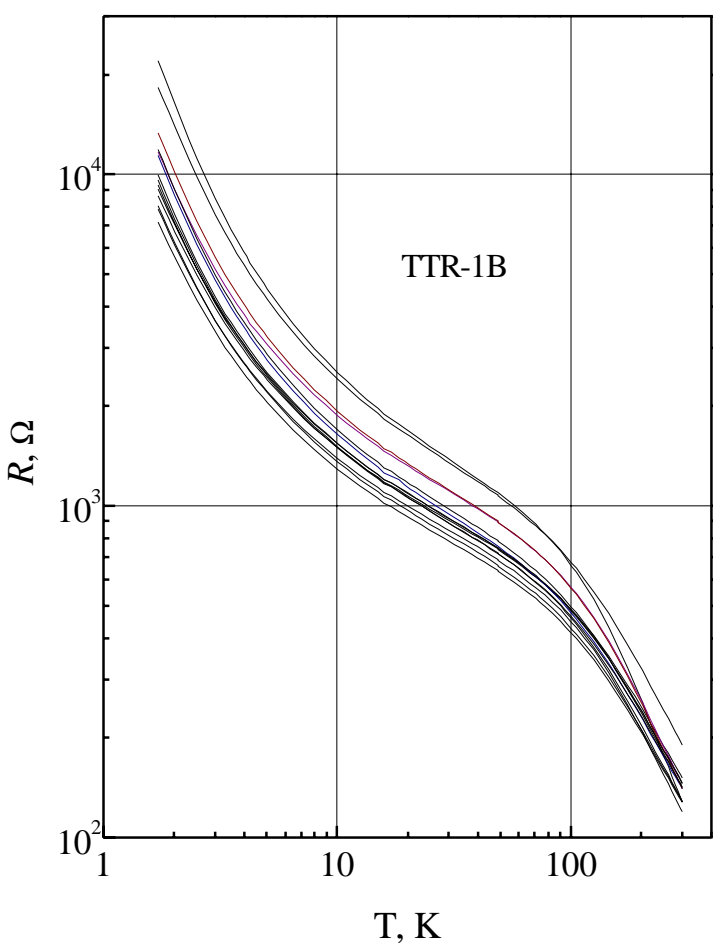

Fig. 5. Thermometric characteristics of the batch of TTR-1B thermometers fabricated from the same thermosensitive $\mathrm{Ge} /$ GaAs structure.

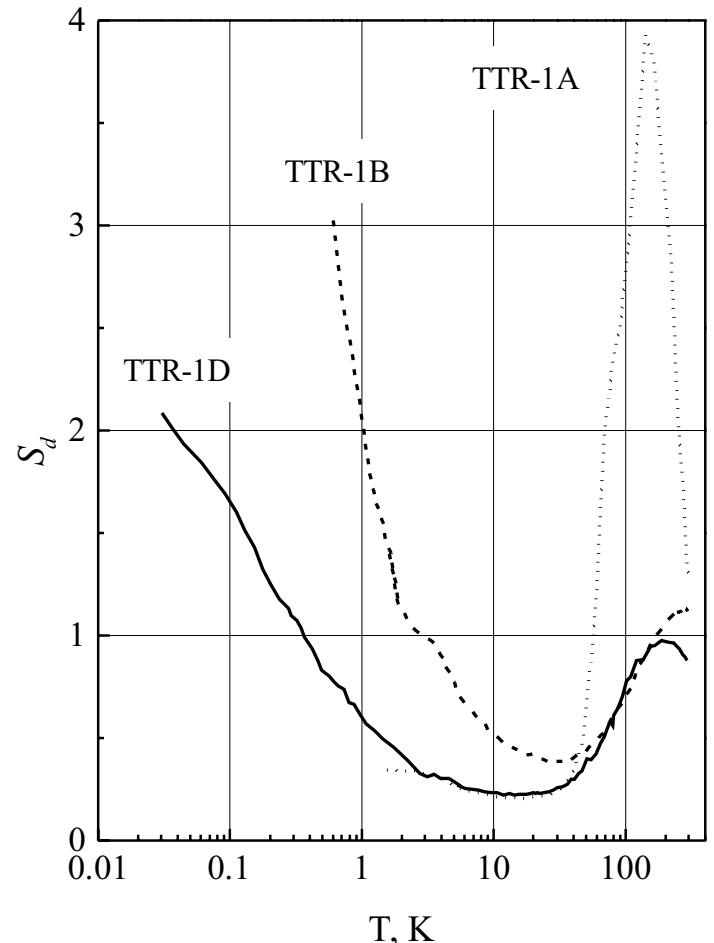

Fig. 6. Dimensionless sensitivity for TTR-1A, TTR-1B and TTR-1D thermometers. 


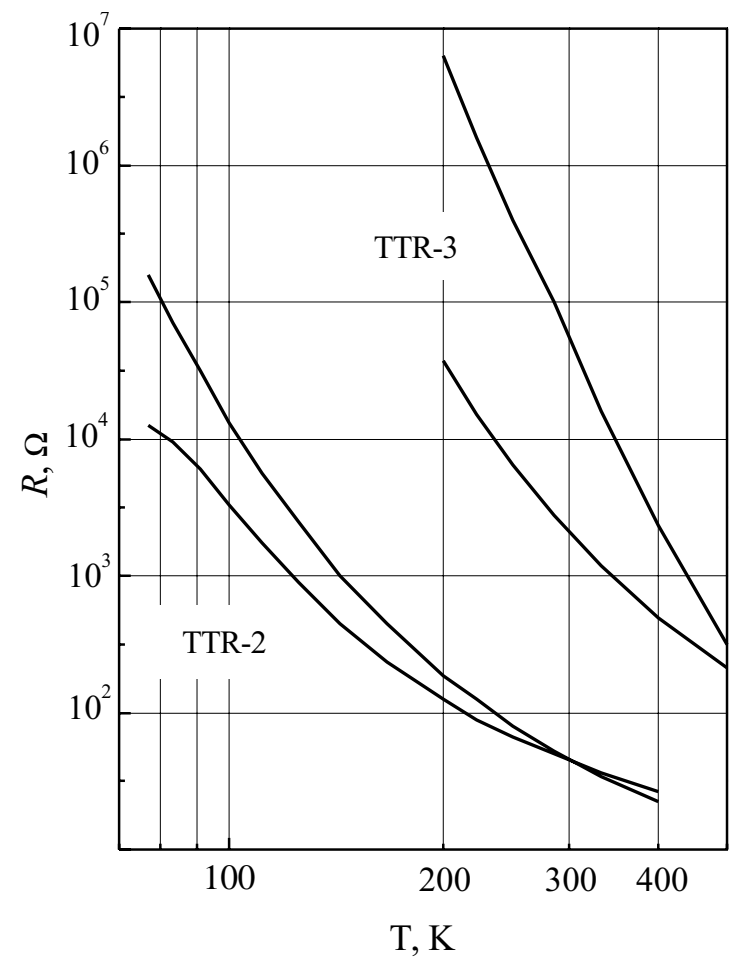

Fig. 7. Temperature dependences of resistance for TTR-2 and TTR-3 thermometers.

\section{Effect of the magnetic field on thermometers}

At present great attention is paid to investigations of behaviour of cryogenic thermometers in magnetic fields, and to development of special thermometers for operation in high magnetic fields. The influence of a magnetic field on a sensor results in errors in temperature measurements. Magnitude of these errors depend on the properties of material from which the thermosensitive element is made and on the nature of the physical effect (in the case of thermoresistor, on the transport mechanism) responsible for the thermal sensitivity.

Consider the effect of the magnetic field on different types of Ge film thermometers in the dependence on its magnitude and temperature. Investigations of TTR-1A and TTR-1B thermometers were carried out in the International Laboratory of High Magnetic Fields and Low Temperatures in Wroclaw, Poland, and studies of TTR-1D thermometers were conducted in the Center for Ultralow Temperature Research at the University of Florida in Gainsville, USA.

The investigations have shown that the effect of magnetoresistivity for the thermometers depends essentially on the conditions of fabrication of Ge films, i.e., on the transport mechanism responsible for the thermosensitivity. Depending on the magnetic field value and temperature, the magnetoresistance for different types of thermometers can be positive or negative. The presence of magnetoresistance effect results in an error of temperature determination. Such an error can be presented as the ratio $\Delta \mathrm{T} / \mathrm{T}(\%)$, where $\Delta \mathrm{T}=\mathrm{T}(B)$ - T, $\mathrm{T}$ is a temperature measured at $B=0$, and $\mathrm{T}(B)$ is some temperature measured in the magnetic field $B$.

In Figs. 8, 9, 10 the dependences of the magnetoresistance $(\Delta R / R)$ on the magnetic field are shown for different temperatures. The orientation of the magnetic field with respect to the sensor is shown in Fig. $2 \mathrm{~b}$. In that configuration the transverse magnetoresistance has been observed in the Ge films $(\overrightarrow{\mathbf{j}} \perp \overrightarrow{\mathbf{B}} \| \overrightarrow{\mathbf{n}}$, where $\overrightarrow{\mathbf{n}}$ is the normal to the film surface, $\overrightarrow{\mathbf{B}}$ and $\mathbf{j}$ are the vectors of the magnetic induction and current, respectively). The magnitudes of errors in temperature determination, $\Delta \mathrm{T} / \mathrm{T}(\%)$, caused by the magnetic field in thermometer readings for some temperatures and magnetic fields are presented in Table 1.

Investigations of the magnetic field effect on characteristics of TTR-1B thermometers were independently carried out in Joint Institute for Nuclear Research, Dubna, Russia and published in [15].

\section{Effect of irradiation on thermometers}

Investigations of the effect of irradiation (gamma-ray and neutron) on thermometers TTR-1A, TTR-1B and TTR-1D were carried out in Joint Institute for Nuclear

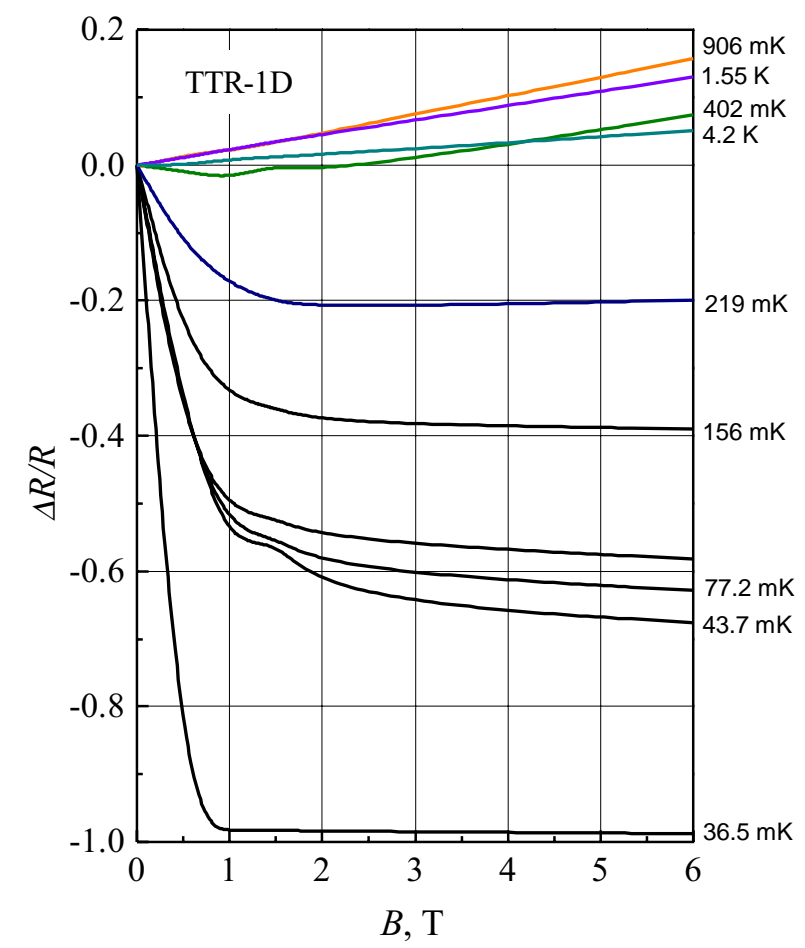

Fig. 8. Dependences of magnetoresistance on the magnetic field for TTR-1D thermometer. 


\section{F. Mitin: Resistance thermometers based on the germanium films ...}

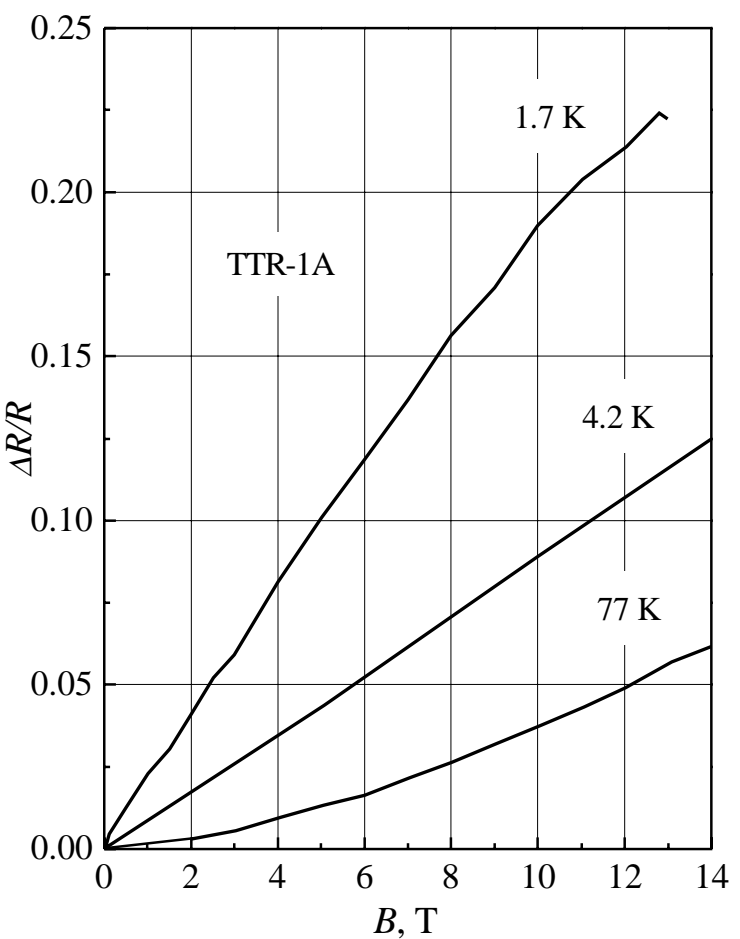

Fig. 9. Dependencies of magnetoresistance on the magnetic field for TTR-1A thermometer.

Research, Dubna, Russia and in CERN, Geneva, Switzerland and published in [15-17]. The experimental conditions and the main results are as follows.

The irradiation of thermometers were carried out at room temperature $(290 \mathrm{~K})$ and in liquid nitrogen

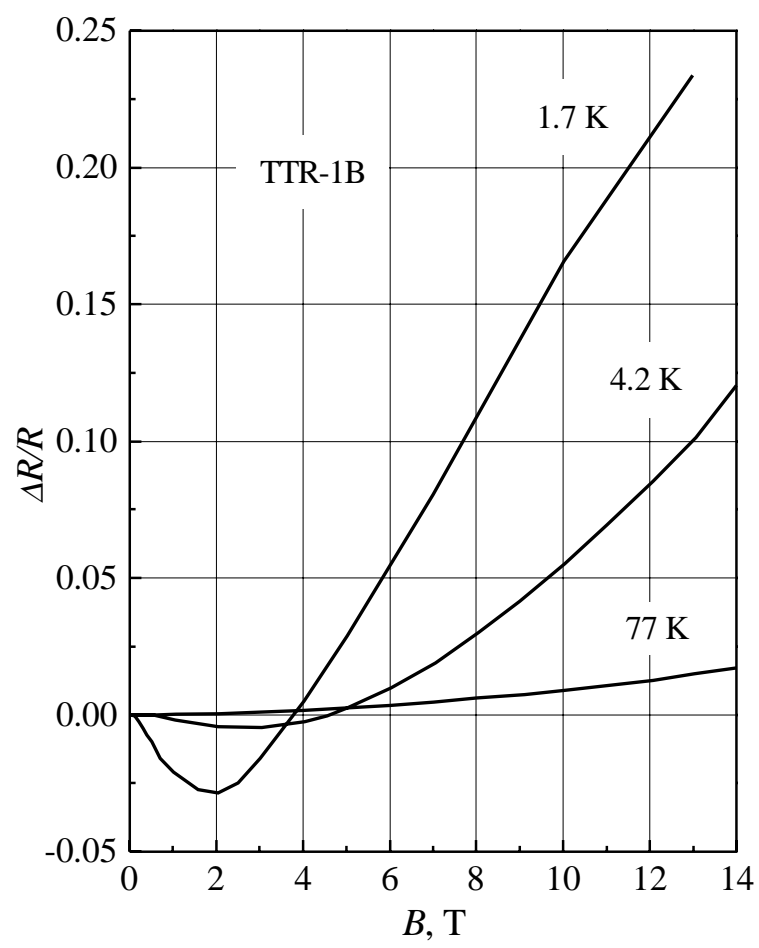

Fig. 10. Dependences of magnetoresistance on the magnetic field for TTR-1B thermometer.

$(77.4 \mathrm{~K})$. As the source of irradiation, the pulse neutron reactor providing the neutron flux with the frequency of $5 \mathrm{~Hz}$ and pulse halfwidth of about $220 \mu$ s was used. The peak energy in the pulse was about $1500 \mathrm{MW}$. The reactor provided the full spectrum of fast neutrons in the range

Table 1. $\Delta \mathrm{T} / \mathrm{T}(\%)$ as a function of the magnetic field and temperature for different models of germanium film thermometers.

\begin{tabular}{|c|c|c|c|c|c|c|}
\hline \multirow{2}{*}{$\begin{array}{c}\text { Thermometer } \\
\text { model }\end{array}$} & \multirow{2}{*}{$\begin{array}{c}\text { Temperature } \\
\text { (K) }\end{array}$} & \multicolumn{5}{|c|}{ Magnetic field (T) } \\
\hline & & 2.5 & 4 & 6 & 8 & 14 \\
\hline \multirow[t]{3}{*}{ TTR-1A } & 1.7 & -18.35 & -28.72 & -41.9 & -55.15 & - \\
\hline & 4.2 & -9.39 & -14.82 & -22.8 & -30.94 & -54.77 \\
\hline & 77.4 & -0.13 & -0.28 & -0.54 & -0.85 & -1.97 \\
\hline \multirow[t]{3}{*}{ TTR-1B } & 1.7 & 1.04 & -0.21 & -2.43 & -4.62 & - \\
\hline & 4.2 & 0.45 & 0.25 & -1.04 & -2.61 & -10.67 \\
\hline & 77.4 & -0.11 & -0.23 & -0.48 & -0.81 & -2.25 \\
\hline \multirow[t]{5}{*}{ TTR-1D } & 0.1 & 63.9 & 66.7 & 69.5 & - & - \\
\hline & 0.2 & 17.0 & 16.7 & 16.2 & - & - \\
\hline & 0.4 & -0.3 & -2.5 & -6.6 & - & - \\
\hline & 1.6 & -8.7 & -13.0 & -18.8 & - & - \\
\hline & 4.2 & -5.0 & -8.1 & -12.0 & - & - \\
\hline
\end{tabular}


from $10^{-1}$ to $20 \mathrm{MeV}$ with mean energy of about $1 \mathrm{MeV}$. The neutron fluence could be as high as $10^{12} \mathrm{~cm}^{-2} \mathrm{~s}^{-1}$. The neutron irradiation was accompanied with the gamma irradiation. The gamma irradiation could be obtained with a maximum dose up to $10 \mathrm{~Gy} / \mathrm{s}$ with mean energy close to $1.5 \mathrm{MeV}$ [15].

In the experiments on irradiation of TTR-1B thermometers at temperature $290 \mathrm{~K}$ the beam of fast neutrons in the range of fluences from $10^{8}$ to $10^{10} \mathrm{~cm}^{-2} \mathrm{~s}^{-1}$ and gamma irradiation of $0.11 \mathrm{~Gy} / \mathrm{s}$ were used. Prior to irradiation, thermometers were calibrated, and after irradiation their characteristics were compared at four temperatures: $1.87,4.24,77.4$, and $287.5 \mathrm{~K}$. The maximum irradiation dose was $2 \times 10^{14} \mathrm{n} / \mathrm{cm}^{2}+3 \times 10^{3} \mathrm{~Gy}$. Simultaneously with investigations of Ge film thermometers TTR-1B the carbonic thermometers of TBO-type were investigated manufactured in VNIIFTRI, Russia. The results of investigations are presented in Table 2 [15]. It should be noted that up to neutron doses of $1.5 \times 10^{13} \mathrm{n} / \mathrm{cm}^{2}$ and that of gamma irradiation of 220 Gy there were no changes observed in characteristics of TBO and TTR$1 \mathrm{~B}$ thermometers.

In the experiments on irradiation of TTR-1A and TBO thermometers at $77.4 \mathrm{~K}$ the beam of fast neutrons with the fluence of $7.5 \times 10^{9} \mathrm{~cm}^{-2} \mathrm{~s}^{-1}$ and gamma-irradiation of $0.11 \mathrm{~Gy} / \mathrm{s}$ were used. The investigation of the sensors was performed in liquid nitrogen during irradiation. TTR-1A and TBO thermometers showed the error in temperature measurements at $77.4 \mathrm{~K}$ less than $\pm 100 \mathrm{mK}$ up to the dose of $1.5 \times 10^{15} \mathrm{n} / \mathrm{cm}^{2}+3.3 \times 10^{4} \mathrm{~Gy}$. After the dose $1.5 \times 10^{15} \mathrm{n} / \mathrm{cm}^{2}$, the system was heated to $290 \mathrm{~K}$, and further irradiation was performed at $290 \mathrm{~K}$. After heating and further irradiation the error in temperature measurements in liquid nitrogen for TTR-1A thermometer increased reaching $1.8 \mathrm{~K}$ at $4.5 \times 10^{15} \mathrm{n} / \mathrm{cm}^{2}+$ $7 \times 10^{4}$ Gy and $2.0 \mathrm{~K}$ at $5.2 \times 10^{15} \mathrm{n} / \mathrm{cm}^{2}+8 \times 10^{4}$ Gy [15].

The investigations of the irradiation effect on TTR$1 \mathrm{D}$ thermometers at $77.4 \mathrm{~K}$ showed that the error in temperature measurements was $\Delta \mathrm{T}<100 \mathrm{mK}$ at doses $10^{14} \mathrm{n} / \mathrm{cm}^{2}$ and $10^{4} \mathrm{~Gy}$. Further irradiation up to the dose of $4 \times 10^{15} \mathrm{n} / \mathrm{cm}^{2}$ led to the error of about $4 \mathrm{~K}$. However, when the irradiation was stopped and the sensors were heated to $290 \mathrm{~K}$, they practically fully restored their characteristics, so that at $77.4 \mathrm{~K}$ the change in resistance as compared to the initial value resulted in the error of about $30 \mathrm{mK}[16]$.

\section{Conclusion}

Germanium films on gallium arsenide can be used for fabrication of small-size resistance thermometers covering the range of operating temperatures from 0.02 to $500 \mathrm{~K}$. The following models of thermometers were designed, fabricated and studied: TTR-1A (1.5-300 K), TTR-1B (0.5-300 K), TTR-1D (0.02-300 K), TTR-2 (50$400 \mathrm{~K})$ and TTR-3 (200-500 K). These thermometers have different temperature sensitivity and different characteristics in magnetic fields.

From the set of low-temperature thermometers the TTR-1B (in the whole range of measured temperatures) and TTR-1D (at temperatures above $0.3 \mathrm{~K}$ ) can be used for the temperature measurements in magnetic fields. The TTR-1A thermometer is not suited for temperature measurements in magnetic fields.

The effect of ionizing irradiation (neutrons and gamma-rays) on the types of thermometers under investigation leads to essential changes in the characteristics $(\Delta \mathrm{T} \geq 100 \mathrm{mK}$ at $77.4 \mathrm{~K})$ only at the neutron + gamma doses greater than $1.5 \times 10^{15} \mathrm{n} / \mathrm{cm}^{2}+3.3 \times 10^{4} \mathrm{~Gy}$.

\section{Acknowledgement}

The author would like to express his gratitude to Prof. M.Oszwaldowski (Politechnika Poznanska, Poznan, Poland), Prof. J. Klamut (International Laboratory of High Magnetic Fields and Low Temperatures in Wroclaw, Poland) and to Dr. I.Yu. Nemish (Special Design Office of ISP NASU, Kiev, Ukraine) for collaboration and assistance in thermometer investigations in high magnetic fields and at low temperatures. The author is also grateful to Prof. G. G. Ihas (University of Florida, USA) for examination of thermometers at ultra-low temperatures $(25 \mathrm{mK}-4.2 \mathrm{~K})$ and in high magnetic fields. The author would like to thank Dr. Yu. P. Filippov (Joint Institute for Nuclear Research, Dubna, Russia) for the interest in germanium film thermometers and investigation of thermometers under the influence of radiation and for helpful discussions. The author is thankful to Dr. N. S. Boltovets (Research Inst. «Orion», Kiev) for collaboration during design of the case, micropackaging and fabrication of thermometers, and to V. V. Kholevchuk (ISP NASU, Kiev) for assistance in conduction of the study.

Table 2. Temperature measurement error after the dose of $2 \times 10^{14} \mathrm{n} / \mathrm{cm}^{2}+3 \times 10^{3} \mathrm{~Gy}[15]$.

\begin{tabular}{|c|c|c|c|c|}
\hline \multirow[t]{2}{*}{ Thermometer type } & \multicolumn{4}{|c|}{ Error in $\mathrm{mK}$ at different temperatures } \\
\hline & $287,5 \mathrm{~K}$ & $77,4 \mathrm{~K}$ & $4,24 \mathrm{~K}$ & $1,87 \mathrm{~K}$ \\
\hline $\mathrm{TBO}$ & -130 & -85 & -12 & - \\
\hline TTR -1B №147 & -530 & -20 & -10 & -10 \\
\hline
\end{tabular}




\section{F. Mitin: Resistance thermometers based on the germanium films ...}

The author thanks Prof. R. V. Konakova and Prof. E. F.Venger (ISP NASU, Kiev) for the support of this research and design work and for helpful discussions.

\section{References}

1. O. A. Gerashchenko, A. N. Gordov, A. K. Eremina et al., Temperature measurements. Reference book, Naukova Dumka, Kiev (1989), 704 p. (in Russian).

2. M. P. Orlova, O. F. Pogorelova, S. A. Ulybin, Low temperature thermometry, Energoatomizdat, Moscow (1987), 280 p. (in Russian).

3. T. Junquera, J. F. Amand, J. P. Thermeau, and J. Casas-Cubillos, Neutron irradiation tests of calibrated cryogenic sensors at low temperatures, Advances in Cryogenic Engineering, 43, pp. 765772 (1998).

4. Temperature Measurement and Control, Catalog Lake Shore Cryotronics, Inc., (1995).

5. D. A. Kichigin, O. A. Mironov, V. V. Mitin et al., Kinetic effects in heteroepitaxial films of p-germanium at low temperatures, Elektronnaya Tehnika, ser. Materialy, 5, p.41-44 (1984) (in Russian).

6. N. P. Garbar, L. A. Matveeva, V. F. Mitin, Yu. A. Tkhorik, R. Harman, Yu. M. Shvarts, and Z. Stroubek, Heavily doped and strongly compensated heteroepitaxial germanium films, Fiz. Tekh. Poluprovodn., 21, No. 3, pp. 393-399, March 1987 [Sov. Phys. Semicond. 21(3), pp. 245-249, March 1987].

7. T. V. Belousova, T. I. Kitaeva, Yu. G. Sadofiev, A. B. Tolstoguzov, Heterodiffusion of gallium in germanium layers on gallium arsenide obtained by MBE technique, Poverkhnost, No.6, p.6065 (1991).
8. A. V. Yeroshkin, I. V. Zakurdaev, Yu. G. Sadofiev et al. Specific features of the epitaxy and properties of $\mathrm{C}^{\mathrm{IV}} / \mathrm{A}^{\mathrm{III}} \mathrm{B}$. heterojunctions, Obz. Po elektron. Tekhnike ser. 2, 3, p.6-15 (1989) (in Russian).

9. V. F. Mitin, Yu. A. Tkhorik and E. F. Venger, All-purpose technology of physical sensors on the base of Ge/GaAs heterostructures, Microelectronics Journal, 28, pp. 617-625 (1997).

10. V. Mitin, Microsensors of temperature, magnetic field, and strain, Cryogenics, 34, pp.437-440 (1994).

11. V. F. Mitin, Ge/GaAs heterostructure: preparation, properties, and application to sensors, Molecular Physics Reports, 21, pp.7178 (1998).

12. V. F. Mitin, E.F. Venger, N.S. Boltovets, M. Oszwaldowski and T. Berus, Low-temperatue Ge film resistance thermometers, Sensors and Actuators A, 68(1-3) pp. 303-306 (1998).

13. V. F. Mitin, Miniature resistance thermometers based on Ge films on GaAs, Advances in Cryogenic Engineering, 43, pp. 749-756 (1998).

14. V. F. Mitin, Miniature temperature sensors based on Ge films, J.Phys. IV France, 8, pp. 193-195 (1998).

15. Yu. P. Filippov, V.V. Golikov, E.N. Kulagin, and V.G. Shabratov, Effects of high intensity cryogenic irradiation and magnetic field on temperature sensors, Advances in Cryogenic Engineering, 43, pp. 773-780 (1998).

16. Yu. P. Filippov, V. V. Golikov, V. M. Miklayev and S. V. Romanov, Tests of the thermometers and cold multiplexers under irradiation, Proc. 17th International Cryogenic Engineering Conference (ICEC17), 14-17 July 1998, Bournemouth, UK.

17. J. F. Amand, J. Casas-Cubillos, T. Junquera, and J. P. Thermeau, Neutron irradiation tests in superfluid helium of LHC cryogenic thermometers, Proc. 17th International Cryogenic Engineering Conference (ICEC17), 14-17 July 1998, Bournemouth, UK. 